\title{
Linx
}

Revue des linguistes de l'université Paris X Nanterre

$62-63 \mid 2010$

Entre syntaxe et discours. Éclairages

épistémologiques et descriptions linguistiques

\section{Eni Puccinelli Orlandi, La Construction du Brésil. À propos des discours français sur la Découverte}

Paris, L'Harmattan, 2011

\section{Sonia Branca-Rosoff}

\section{OpenEdition \\ Journals}

Édition électronique

URL : http://journals.openedition.org/linx/1387

DOI : $10.4000 /$ linx.1387

ISSN : 2118-9692

Éditeur

Presses universitaires de Paris Nanterre

\section{Édition imprimée}

Date de publication : 1 juillet 2010

Pagination : 197-199

ISSN : 0246-8743

Référence électronique

Sonia Branca-Rosoff, «Eni Puccinelli Orlandi, La Construction du Brésil. À propos des discours français sur la Découverte », Linx [En ligne], 62-63 | 2010, mis en ligne le 04 décembre 2013, consulté le 22 septembre 2020. URL : http://journals.openedition.org/linx/1387 ; DOI : https://doi.org/10.4000/linx. 1387 


\section{Compte rendu}

Eni PUCCINELLI ORLANDI, La Construction du Brésil. À propos des discours francais sur la Découverte, Paris, L'Harmattan, 2011.

Nous devons à la forte exigence théorique d'Eni Orlandi, professeure d'analyse du discours au Brésil, un livre passionnant tant par l'objet dont il traite, les discours français sur la découverte du Brésil ou plus largement les rapports entre les Européens et les terres (sud)-américaines qu'ils ont découvertes, que par les outils théoriques mis en ouvre pour aborder cette histoire, outils dont l'ensemble constitue une version brésilienne de l'AD ${ }^{1}$ qu'E. Orlandi a largement contribué à élaborer et à diffuser. Un certain nombre de chapitres du livre reprennent des articles publiés entre 1984 et 1988, mais ils sont ici remis dans une perspective d'ensemble qui permet d'apprécier la cohérence et l'importance du courant de pensée représenté par le courant brésilien d'analyse du discours.

Le premier but de cette analyse des discours français sur le Brésil est de déconstruire les visions culturalistes d'une «identité brésilienne » réduite à quelques clichés exotiques, en soulignant à rebours combien le rapport à l'«autre»- en particulier l'Occidental - est au cœur des constructions identitaires des Brésiliens et en inscrivant cette relation non dans une simple chronologie, mais plutôt dans un parcours de ses interprétations successives. Le livre est bâti sur de fortes dichotomies entre histoire et culture ; chronologie et historicité des interprétations ; sujet et sujet du langage, sens et silence. Si l'intérêt de l'AD pour les conditions de production, pour l'ancrage énonciatif et historique des interlocuteurs qu'E. Orlandi met en œuvre dans

\footnotetext{
${ }^{1}$ Il s'agit du modèle dit «français » d'analyse du discours, élaboré par M. Pêcheux et les chercheurs de son groupe, notamment J.-J. Courtine. On note aussi la forte influence du M. Foucault de Surveiller et Punir dans l'intérêt pour les liens étroits entretenus entre discours de savoir et techniques de pouvoir (p. 9). E. Orlandi écrit dès les premières pages que «voir, rendre visible est une forme d'appropriation » et un peu plus loin que « découverte signifie alors conquête » (p. 13).
} 
son ouvrage est bien connu ${ }^{2}$, le silence et le silenciement sont des apports propres qu'on lui doit.

Dans la catégorie du silence, elle distingue des silences «locaux » et le silence « constitutif», « la part du sens qui est nécessairement sacrifiée, effacée, quand on dit» (p. 51), puisque dire X c'est forcément ne pas dire Y. Ainsi dire «le Brésil a été découvert» selon le point de vue occidental, c'est ne pas dire «le Brésil a été envahi», ce qui pourrait être le point de vue des autochtones. Le silence local est plus directement politique, c'est par exemple celui de la censure (Voir E. Orlandi, Les formes du silence, Edition des Cendres, Paris, 1994 et le chapitre 4 de cet ouvrage).

Le livre traite deux thèmes entremêlés, celui des discours sur l'Indien et celui des discours sur la nation brésilienne. Les récits des voyageurs et des missionnaires produits entre les $\mathrm{XVI}^{e}$ et $\mathrm{XIX}^{e}$ siècles ${ }^{3}$ ont peu à peu contribué à rendre l'Indien observable - ce qui assure un premier degré de maitrise sur lui : dans la période qui suit la « découverte » du Brésil, les langues indiennes commencent à être abordées dans les récits des voyageurs, mais ces derniers n'en retiennent qu'une suite d'énoncés correspondant à des situations stéréotypées et caricaturales ${ }^{4}$. Certaines de ces langues seront ensuite décrites une fois pliées aux catégories latines par les missionnaires qui «inventent» des systèmes simplifiés et systématisés à des fins d'évangélisation. Cette mise par écrit prétend rendre compte des langues indiennes, mais c'est au prix de l'effacement de leur altérité liée à l'oralité (p. 163). L'Indien est enfin envisagé comme assimilable, ce qui permet d'oublier son existence comme part autonome de la nation brésilienne (voir notamment p. 59 et sq.). La situation actuelle n'est guère améliorée : E. Orlandi montre que même les linguistes parce qu'ils conduisent à prendre la langue comme critère essentiel de l'existence d'un peuple, contribuent malgré eux à nier les droits des Indiens en tant que peuple. L'exemple du pataxó et de son dialecte hảhảhai - langues quasi éteinte de l'Etat de Bahia - montre qu'en privilégiant les critères linguistiques on prive les Indiens de leur identité. « Acculturés », ils perdent leurs droits de minorités, en particulier leurs droits sur la terre. L'analyse du discours, parce qu'elle s'intéresse moins aux structures des systèmes linguistiques qu'à des productions discursives empiriques, peut donner un statut aux quelques fragments de discours encore connus par des Indiens pataxó pour qui ces vestiges signifient encore quelque chose. Elle peut souligner la manière spécifique qu'ont ces Indiens d'être brésiliens. L'AD offre par conséquent un appui à tous ceux qui entreprennent de déconstruire le discours de la colonisation.

Le silence fait sur les Indiens a aussi des conséquences pour la nation brésilienne et la langue «nationale ». Les grammairiens ont marginalisé autant que possible l'histoire des échanges entre langue brésilienne en formation et les langues

\footnotetext{
${ }^{2}$ On mentionnera aussi le surprenant concept de sujet qui émerge de l'analyse d'un mythe assurini et qui rappelle les discussions sur le sujet dispersé de Foucault et sa critique de la notion d'auteur.

${ }^{3}$ Le plus célèbre de ces récits est celui de Jean de Léry dont le Voyage à la Terre du Brésil (1580) a été repris et «modernisé » en 2001 par Jean-Christophe Ruffin sous le titre Rouge Brésil.

${ }^{4} \mathrm{E}$. Orlandi aurait pu évoquer les «colloques » pédagogiques destinés à l'apprentissage des langues étrangères depuis le Moyen Age qui ont pu servir de modèles, sans que la caricature soit liée à une perception péjorative.
} 
indigènes; ils ont négligé les traces que laissent les parlers indiens dans la langue «fluide » (la langue quotidienne), et ils ont imposé une conception largement imaginaire d'une langue «une». E. Orlandi cite ainsi des traits du tupi qui ont influencé le portugais brésilien et qui sont minorés comme le suffixe -rana, modalisateur signifiant « comme si c'était» : par exemple, dans la région Nord, «netarana » signifie « comme si elle était ma petite fille». La vision unitaire empêche en tout cas les Brésiliens de sortir du face à face Brésil/Occident, à peine troublé par les traces de l'esclavage avec l'arrivée massive des Noirs.

Pour un Français, il est particulièrement stimulant de voir ce que produisent en 2011 les développements brésiliens de la pensée de Pêcheux et de les comparer aux analyses françaises qui se réclament de l'AD. Lorsqu'ils ne se sont pas ralliés aux interactionnistes, les Français sont allés vers un travail de plus en plus fin sur le sens porté par les formulations du discours, alors que l'AD dans la conception d'E. Orlandi met l'accent sur «l'historicité du discours au sens large » et affirme "qu'il faut savoir être linguiste et oublier qu'on l'est» (Orlandi et Guimares 2007 p. 42) $)^{5}$. Il est significatif que ce livre qui défend une analyse du discours ouverte sur l'histoire - et qui dialogue de façon critique avec les développements théoriques français soucieux d'analyse interne de la langue au détriment de l'intervention critique dans le champ social - ait lieu à propos d'une présentation du regard occidental sur le Nouveau Monde et la façon dont les Brésiliens peuvent aujourd'hui en décrire les effets de domination afin de les déplacer.

\section{Sonia BRANCA-ROSOFF Paris3-Clesthia Syled}

\footnotetext{
5 Orlandi Eni P. et Guimaraes Eduardo, 2007, Un dialogue atlantique. Production des sciences du langage au brésil, Lyon, ENS éditions.
} 